\title{
Determinants Influencing Bed Occupancy Rate (BOR) at Langsa Hospital in the Covid-
} 19 Pandemic

\author{
Iskandar $^{1}$, Arifah Devi Fitriani ${ }^{1}$, Asriwati ${ }^{1}$ \\ ${ }^{1}$ Public Health Sciences, Faculty of Public Health, Helvetia Institute, Indonesia \\ Received: September 15, 2021 \\ Received in Revised: October 24, 2021 \\ Accepted: November 4, 2021
}

\begin{abstract}
Health facilities with referral status for COVID-19 cases, reported a decrease in non-COVID inpatients, compared to facilities with non-referral status. The decrease in bed utilization (BOR) was very significant in several health facilities including Langsa Hospital 10-15\% before $65-75 \%$,. This study aims to study the Determinants of Factors Affecting the Decrease in Bed Utilization (BOR) at Langsa Hospital during the COVID-19 Pandemic Period in 2021. The research approach uses descriptive qualitative data collection techniques through in-depth interviews and informants are determined by purposive sampling of key informants, namely 4 inpatients and 2 supporting informants. The total number of informants is 6 people. The research instrument is the researcher himself and data collection through interviews, documentation and field observations. Data analysis technique with taxonomy. The results of the study, namely the availability of services due to reduced facilities, hospital policies in implementing health care programs, priority to hospitals from patients in independent practice health care services and community stigma regarding the confirmation of COVID-19 status in each patient, were the driving factors for the decrease in BOR at Langsa Hospital in the 2021 COVID-19 pandemic. It is recommended that the Langsa Hospital management coordinate with IPSRS for the availability of facilities, the health promotion section conducts more effective promotion and socialization methods and nursing provides training on effective communication.
\end{abstract}

Keywords: BOR, Covid-19, Service Availability, Policy, Priority, Stigma

\section{Introduction}

Ignorance of the COVID-19 pandemic has led to the formation of a wrong stigma in perceiving the epidemic situation. This situation is supported by the dissemination of inaccurate information which results in disproportionate public actions. Stigma mostly stems from excessive fear that is believed to be the truth and is reinforced by media such as cyberspace through the broadcast of various events. This is exacerbated by health promotion from related agencies that has not been optimal in the speed of providing information about COVID-19 to the public. The search found 2,311 stigmas, rumors, conspiracy theories in 25 languages in 87 countries in the world that led to the formation of inaccurate perceptions in society.

The reduction in BOR in some countries can be caused by a policy re-arrangement that limits visits to several rooms in hospitals, especially critical rooms. Strict regulations are aimed at minimizing cases of COVID-19 and preventing its spread in the community. The restrictions were accompanied by rearrangements in the way patients were received so that some medical personnel reported a significant reduction in activities related to procedures to patients, including surgery (Gilmartin, 2004).

Countries where patient visits have not decreased in the long term due to proper policy making so that the situation is controlled and patient visits return to normal within 17 weeks after the

Copyright @ 2021, Journal of Asian Multicultural Research for Medical and Health Science Study, Under the license CC BY-SA 4.0 
first wave of COVID-19 hit the region. stones in the urinary tract, so that the pandemic does not prevent patients from visiting health facilities.

Health facilities that have the status as a referral place for COVID-19 cases, reported a decrease in non-COVID inpatients, compared to facilities with non-referral status. This is related to the public's reluctance to use facilities with a high probability of being infected with COVID-19. The decrease in visits showed a significant number of $69.3 \%$ at referral hospitals, while facilities that were not involved in handling COVID-19 experienced a lower decline of around $30 \%$ (Thekkur et al., 2021).

The Langsa Regional General Hospital (RSUD) is one of the COVID-19 referral health facilities in Aceh Province. This facility before the 2019 pandemic had a BOR of around 60$75 \%$. The BOR, like other regions with a pandemic, has decreased significantly to $10-15 \%$ after the pandemic. From April to December 2020, the overall BOR of the Langsa Hospital which was reported was recorded at $10-15 \%$. BOR in critical space, has the same description. The Intensive Care Unit (ICU) after the pandemic has a BOR of around 30-40\%. This situation is not the same as the rooms dedicated to COVID-19 sufferers with high BOR, such as the New Emerging and Re-Emerging Infectious Diseases (PINERE) and Negative Pressure Isolation Rooms (RITN).

Interviews that the author conducted with 10 patients who visited Langsa Hospital got an overview of 8 patients stating that they were afraid to visit the hospital unless it was an urgent situation. Patients are afraid of contracting COVID-19, which they know is a very dangerous disease.

The researcher saw that there were variations that occurred in several central hospitals as COVID-19 referrals and regional hospitals regarding the percentage of BOR, but the author felt the need to extract deeper data related to the factors that affect the percentage of BOR in a concrete way, because of this, the author is interested in conducted a study on the determinants of the factors that affect the use of BOR beds in Langsa Hospital during the 2020 COVID-19 pandemic. This study aims to obtain qualitative information about the determinants of factors that affect the decrease in bed utilization (BOR) in Langsa Hospital. During the COVID-19 pandemic in 2021

\section{Methods}

The type of research used is qualitative research with descriptive analysis. This research was conducted at Langsa Hospital. The time of research on the determinants that affect the use of BOR beds at Langsa Hospital during the Covid-19 pandemic has been carried out in July 2021. The number of informants in this study was determined purposively, namely 6 people. The informants that the researchers used as data sources consisted of key informants of patients who visited Langsa Hospital and were required to be hospitalized, as well as supporting informants for the Head of Nursing, Deputy Director of Services. There are 4 key informants while the supporting informants are 2 people, each consisting of the Head of Nursing and 1 Deputy Director of Services. Researchers are looking for informants by determining their own characteristics that the author considers will be able to support the collection of representative data regarding the influence of BOR from external factors so that a broader picture can be obtained regarding the phenomenon of decreasing BOR during the COVID-19 pandemic

\section{Results and Discussion}

\section{Service Availability}

Based on the results of research conducted at Langsa Hospital on 6 informants, it is known that the role of service availability in reducing bed utilization is very large. The availability of

Copyright @ 2021, Journal of Asian Multicultural Research for Medical and Health Science Study, Under the license CC BY-SA 4.0 
services that are not in accordance with the patient's needs is an important part that drives a decrease in the use of beds/BOR at Langsa Hospital. Patients expect to get regular visits from experts, in addition to fulfilling the desire to be treated in a room with facilities as expected. Suspicion will be ignored making the patient reluctant to be hospitalized. Patients feel calm when they get services from experts according to their needs because they believe that their disease will be overcome compared to others (Sun et al., 2020). Another disturbing feeling of not being fulfilled in this aspect is the lack of comfort due to mingling with other patients, including having a place for cleaning needs that can be used alone and close proximity when needed. Like RH who feels calm when he has been treated by a specialist and SH who feels comfortable with a separate room and has a bathroom.

Availability of services is one of the hospital's internal factors that affect BOR and define this aspect whenever needed, such as the availability of medical personnel and paramedics whenever needed and the availability of types of services. Medical personnel are doctors who treat inpatients while nursing personnel are nurses who work in hospitals to treat inpatients (Needleman et al., 2011; Van Zyl \& Rheeder, 2008).

Other things that must be provided by hospitals for patients who use health services include services in the form of availability of facilities. Facilities and infrastructure that must be available, especially in VIP inpatient rooms, other than patient beds, are cupboards, nurse calls, tables, chairs, televisions, separating curtains if any, lighting, ventilation and sofas. The bathroom is an integral part of the room for patients. The availability of the things that have been described greatly affects the utilization of hospital beds as stated by Harold Koening HFZ from the internal aspect of a health facility.

The role of the availability of health services on the use of beds in Langsa Hospital, is illustrated by the statements given by several informants such as that expressed by SH that he feels calmer when experts examine his illness. Not a substitute general practitioner. The informant heard this from a neighbor who had to be hospitalized during the COVID-19 pandemic. This, according to the researcher, shows that although it has been informed that the treatment provided by substitute medical personnel is a referral from expert medical personnel, it cannot increase patient satisfaction with the services provided. Patients want specialist doctors who understand their illness who come to check and visit in the inpatient room every day. Intermittent visits are perceived by patients as not meeting their needs when hospitalized, so that they feel that using a hospital bed is a futile act, as is the assumption of KN.

The aspect of service availability that was felt to be lacking during the COVID-19 pandemic was the reduction in facilities as before the disease hit. The informant, who had been hospitalized before the pandemic, stated that cold room facilities were hard to come by. This is related to the use of rooms for COVID-19 patients. This complaint, according to researchers, is because the patient wants to feel comfortable while being treated. Additional difficulties such as having to share a room with other patients and meeting the need for cleaning are difficult, making this an unpleasant experience. A bad experience is a situation that no individual wants to repeat and in the case of having to be hospitalized, patients will look for facilities other than Langsa Hospital as stated by RH.

The assumption is circulating that the handling of patients in inpatient rooms is mostly done by substitute general practitioners and the reduced availability of facilities due to the COVID19 pandemic has caused the people of the Langsa City area to feel reluctant to come to take advantage of services to Langsa Hospital even though they have health problems. Patients mostly use other health services other than Langsa Hospital and avoid having to be hospitalized. This action led to a decrease in the number of hospitalized patients which resulted in a decrease in the utilization of available beds in hospitals from before the pandemic. This

Copyright @ 2021, Journal of Asian Multicultural Research for Medical and Health Science Study, Under the license CC BY-SA 4.0 
accumulation over a period of one year showed a significant decrease in the BOR at Langsa Hospital.

This is in accordance with research that found the service availability factor as an aspect that was described as having a high influence on the utilization of hospital beds/BOR. Patients who have health problems and want to be resolved immediately by visiting a tertiary care place, make this item a top priority for deciding on hospitalization (Saaty \& Peniwati, 2013). This is in accordance with research which states that the fulfillment of aspects of quality health services will increase the occupancy rate of a hospital. Quality refers to the availability of services in accordance with patient expectations, both from the physical aspect of the building to nursing and medical services (tangibles). Patients who are satisfied with the services provided will use the service repeatedly to the same health facility (Cho et al., 2004; Sitzia \& Wood, 1997).

The same description was put forward regarding the relationship between the completeness of human resources, facilities and infrastructure with the hospital BOR. Patients want sufficient resources to serve patients, including facilities. Incomplete means reduce patient satisfaction. Likewise with human resources that provide services from medical and nurses. Insufficient experts needed, have an impact on the waiting time for patients to get services, so that the interest in returning to the hospital decreases (Forster et al., 2003).

Outbreaks or emergencies cannot be the main reason for not meeting patient needs optimally. The existence of limited human resources and facilities due to emergency cases, should not have occurred if proper calculations were made including anticipating this condition. A room containing a bed with the intention of being reserved for emergencies does not have to have a nurse. Planning must be done in advance so as not to make health facilities reduce patient comfort (Sudore, \& Fried, 2010).

\section{Hospital Policy}

Based on the results of research conducted at Langsa Hospital on 6 informants, it is known that the role of hospital policy in reducing bed utilization is significant. Hospital policies during the COVID-19 pandemic that were not understood by patients were one of the causes of the decrease in the use of beds/BOR at Langsa Hospital. The patient feels burdened by the limitation of the number of family accompanying him in the inpatient room, including those who want to visit. The obligation to use masks in the inpatient room for families who care for them, including those who complain about policies. It is felt that the hospital enforces rules that are unpleasant and tend to be difficult, as TA, one of the informants, complained.

The policies taken by Langsa Hospital during the COVID-19 pandemic are inseparable from government regulations. The protocol for handling COVID-19 has been socialized and through the COVID-19 task force, the acceleration of overcoming this outbreak has been carried out, with the hospital as one of the implementers. The enforcement of the current situation as a disaster, based on Law no. 24 of 2007 concerning Disaster Management. The reference in deciding actions to accelerate the prevention of this epidemic is supported by Law No. 4 of 1984 concerning Outbreaks of Infectious Diseases and Law No. 6 of 2018 concerning Health Quarantine. According to Harold Koening HFZ, the policy of a health service facility, such as a hospital, has an influence on the use of beds. This aspect is known to be one that patients consider in making hospitalization decisions (Robinson \& Thomson, 2001).

The COVID-19 pandemic requires health facilities such as the Langsa Hospital to implement health protocols to prevent transmission. Researchers saw patients who were unfamiliar with these regulatory changes perceived preventive measures as a barrier to activity. The use of masks makes it more difficult to breathe and speak, moreover, it must be done throughout the

Copyright $\odot$ 2021, Journal of Asian Multicultural Research for Medical and Health Science Study, Under the license CC BY-SA 4.0 
day like a patient companion in an inpatient room. This is like SH's expression, that the age of the wife who takes care of herself is old so wearing a mask is very disturbing. There is a limit for waiting patients, including what is perceived as a difficulty. The absence of families who can take turns when others buy something makes some needs that must be met, postponed until someone comes. Patients also feel that their family or neighbors who want to visit are limited, even though being visited when sick is a pleasant thing. A visit that cannot be done by many people at once, causes those who want to visit to cancel their intention because they are not used to entering the inpatient room alternately. Finally, the closest people to the patients who participated did not agree to be hospitalized at Langsa Hospital, including people in the living environment as narrated by TA. These various urges continue to be the cause of the unwillingness of patients to be hospitalized and optimally avoid visiting even though they are sick.

The reluctance expressed by the patient above to take advantage of Langsa Hospital when experiencing pain, especially to get hospitalized, has caused many of the available beds in the inpatient room to be unused. This prolonged situation causes the number of hospitalized patients for at least 24 hours compared to the facilities available for a period of one year, which will represent a low percentage of BOR.

The influence of this aspect on patient decisions is in accordance with research conducted on both aspects, in most hospitals in the Taiwan area related to the COVID-19 pandemic, namely limiting visits to visit patients. The new policy was taken with several adjustments, such as visits to non-emergency rooms and non-COVID-19 patients, families only being allowed to visit one ward per day, each patient being visited by two families per day and implementing health procedures. Other things that were investigated before visiting were checking body temperature, family origin of residence (cluster), travel history, contact history, and work. Updates regarding hospital visits with procedures at a more extreme level were taken by several other hospitals, namely no visits at all. This means that no family is allowed to see their sick family in the hospital, until it is time to go home. Restrictions in various ways have led to a decrease in hospital visits including the utilization of services except emergency departments (Gill et al., 2000).

Another study revealed the same thing that restrictions on visiting hospitals during the COVID19 pandemic, as a policy taken in hospitals in Australia to prevent the development of infection clusters from these places, had an impact on the utilization of hospital beds. The restrictions were followed by encouragement to optimize the media, namely telehealth as a forum for conducting health consultations. The majority of users are known to be old, female, have a high level of education and have comorbidities. The use of media is supported because individuals who experience health problems still have to find solutions to the problems they face. The COVID-19 pandemic has caused many people to be reluctant to go to hospitals or use clinics, apart from fear of being exposed to infection as well as various restrictive policies implemented. Tighter visiting regulations cause patients who have to use the media to feel more comfortable using telehealth than going to the hospital. Other patients even said that their consultation with a health professional was more meaningful with this medium than face-toface (Atherton et al., 2018).

\section{Priority to Hospital}

Based on the results of research conducted at Langsa Hospital on 6 informants, it is known that changes in priorities in the utilization of health facilities are the reason for reluctance to come to the hospital. This is related to the COVID-19 pandemic and the status of Langsa Hospital as one of the facilities that treat people with the infection. Patients prefer to buy drugs at the depot, while others choose to visit a health center that has a practice in the vicinity of their residence 
or who has become a subscriber. The avoidance of health facilities such as Langsa Hospital was carried out in various ways, including increasing the frequency of using herbal medicines as did the RH informant. There is a feeling of fear and discomfort in visiting the hospital and it is only done out of necessity, as TA said.

Priority refers to behavior that prioritizes one thing over others, while the behavior of using health services is the use of service facilities provided either in the form of outpatient care, inpatient care, home visits by health workers or other forms of activity from the use of these services based on availability and continuity of services, public acceptance and fairness, easily accessible by the community, affordable, and of good quality. This can be interpreted that the priority in the use of health services can be interpreted as an action to use one health service more often than another.

The difference in the frequency of use can be due to several factors such as knowledge, attitudes, beliefs, perceptions (called the through and feeling factor), resources (resources), people who become references (personal reference), and culture (culture). Another review states that the priority of health service utilization based on this behavior is related to the use of health services which are interpreted as predisposing characteristics, enabling characteristics and need characteristics (Weller et al., 2003).

Priority shift in utilizing types of health services during the COVID-19 pandemic, which previously had a higher frequency of people with health problems coming to the hospital to using other facilities more often. Patients more often come to the drug depot if they have minor health problems or to the health care provider and private practice midwife. A visit to the doctor is also the patient's next choice compared to having to go to Langsa Hospital. Patients prefer to use this facility because they feel safe and comfortable. There are no rules and restrictions that are considered troublesome. Visiting the hospital is only done when experiencing urgent health problems such as severe shortness of breath due to asthma, as stated by KN.

Information with the same focus from all informants, namely placing visits to hospitals, especially Langsa Hospital as the last order or in a position of having no other choice to cure the illness, caused the utilization of beds in the inpatient room to be very low during the COVID-19 pandemic. This can be seen from the 2019 BOR which reached 60-75\%, while calculations made from April to December 2020 after the COVID-19 pandemic only ranged from $10-15 \%$.

Research results in several developed countries have found that priority aspects have an influence on BOR. The COVID-19 pandemic has caused a shift in the utilization of health facilities. The patient does not place the hospital as the main place to cure his illness but shifts to private consultation through the media. The shift in how to get treatment in developed countries that are different from developing countries still illustrates the need for a review of priorities in utilizing health facilities. Germany, as one of the developed countries, experienced the same thing during the pandemic, namely a decrease in inpatients and outpatients. The reduction of more than $50 \%$ includes the cancellation of consultation appointments to see specialist doctors in private practice. Residents of these developed countries are encouraged to use technology when experiencing health problems to get solutions from doctors who have been appointed to serve consultation facilities with the media (12). The shifting priority to get health services during the COVID-19 pandemic is a real impact of various restrictions that cause patients to choose other ways to continue to deal with health problems other than going to the hospital.

The results of the same study suggest that the pandemic has caused a decrease in the utilization of health facilities, both hospitals and visits to specialist doctors who practice privately. The 
decrease in overall hospitalizations is estimated at $46.1 \%$ while visits to private practice doctors reached $66.2 \%$ compared to the same time before the pandemic in 2019. These non-COVID19 patient visits have never reached the same quantity although the end of the lockdown imposed by the government in Northern Italy. The pandemic has made health consultations using the media a priority because they can encourage adherence to social distancing. Health care through cellular is a form of long-term health service that has the hope of being able to reduce the impact of the COVID-19 pandemic on humans (Kennelly et al., 2020).

\section{Stigma}

Based on the results of research conducted at Langsa General Hospital against 6 informants, it is known that the stigma about Langsa Hospital in handling COVID-19 patients in the community is a strong incentive for patients not to use beds in this institution or to be hospitalized. The assumption that examinations to classify patients as COVID-19 or nonCOVID-19 are not necessarily accurate and that every patient is likely to be detected by COVID-19 is some of the stigma that reduces patient interest in Langsa Hospital. The patient heard that the visible symptoms were not as well known as COVID-19 and was in fact diagnosed with the disease, supporting the allegation of arbitrariness in the reading of laboratory results, such as the TA narrative.

Stigma is the extreme disapproval of a person or group of people based on certain characteristics that distinguish them or their existence to be unwanted in society. Stigma is also a set of negative beliefs that a person has to underlie the injustices that a group of people have about something

Stigma is related to social life which is usually directed at people who are seen as different, such as being victims of crime, poverty, and people who are sick. People who are stigmatized are labeled or marked as guilty. Social stigma in the context of health is a negative association between a person or group of people who share certain characteristics and diseases. Outbreak conditions related to social stigma can be interpreted as people being labeled, stereotyped, discriminated against, treated differently, and/or experiencing a loss of status because they are considered to be related to a disease. Stigma is part of the social condition of the community which has been known to have an influence on the utilization of beds in health facilities.

There is a stigma in patients that causes them to be reluctant to take advantage of health services at Langsa Hospital, as stated by TA, that sick people who go to Langsa Hospital are often diagnosed with COVID-19, as stated by informant $\mathrm{SH}$, that neighbors who are known to often get sick When he was admitted to the hospital during a pandemic, he was immediately declared confirmed COVID-19. According to the researcher, this is a description of the absence of public efforts to check the truth of the news. The incident that circulated was immediately believed to be true. News about the treatment of patients upon admission to the hospital circulated widely in social groups and no one could prove the truth or attempt to come to the facility in order to confirm.

The decrease in public interest in using inpatient services at Langsa Hospital because of the stigma, caused many beds for patients to be unfilled. This low interest has continued during the COVIID-19 pandemic since April 2020 so that the accumulation carried out until December 2020 saw a very significant decrease in bed utilization or BOR. The accumulation during this time period illustrates the magnitude of the impact of the pandemic from the stigma aspect so that only a small number of people come to use the beds in the Langsa Hospital inpatient room.

This is in accordance with research publications which state that the decrease in emergency room visits to every hospital in the Netherlands every day is $18 \%$ compared to 2019 before the COVID-19 pandemic occurred. Patients who received hospitalization overall experienced an 
increase but higher in patients with COVID-19 compared to non-COVID-19 patients. The decline was caused by many factors, but the factor of excessive fear of health facilities, such as hospitals, was the main one. The fear is exaggerated because this disease is something new and the role of the media is added

Stigma, such as the description of the results of research on the term, is known to occur in health facilities, especially in dangerous diseases. This action can be directed at health workers or patients who have the disease. Labeling that results in discriminatory actions and subsequent loss of status from those experiencing these actions. Stigma in health facilities has an impact on weakening diagnosis, treatment, and the success of a treatment action. Part of the achievement of quality health services so that everyone who takes advantage of optimal health facilities to recover, it is necessary to take action to eliminate stigma

The results of other studies show the effect of stigma on various sectors in health services, one of which is health workers. Stigma against health workers, especially those who work in hospitals to quarantine confirmed COVID-19 patients, is higher than those who do not work in hospitals. This illustrates that the workplace is one of the causes of the development of stigma in society. Isolation as part of the stigma due to the COVID-19 pandemic is carried out in all parts related to this outbreak. Avoidance by the community is carried out on health workers and their families, including health facilities known to accept COVID-19 patients. This must get the right treatment because stigma causes delays in people coming to health facilities due to exaggerated fears

\section{Conclusion}

The availability of services has an influence on decreasing bed utilization (BOR) at Langsa Hospital during the 2021 COVID-19 pandemic. Policies have an influence on decreasing bed utilization (BOR) at Langsa Hospital during the 2021 COVID-19 pandemic. Priority to Hospitals have a role in reducing bed utilization (BOR) at Langsa Hospital during the COVID19 pandemic in 2021. Stigma has a relationship with decreasing bed utilization (BOR) at Langsa Hospital during the COVID-19 pandemic in 2021

\section{References}

Atherton, H., Brant, H., Ziebland, S., Bikker, A., Campbell, J., Gibson, A., ... \& Salisbury, C. (2018). The potential of alternatives to face-to-face consultation in general practice, and the impact on different patient groups: a mixed-methods case study. Health Services and Delivery Research, 6(20).

Cho, W. H., Lee, H., Kim, C., Lee, S., \& Choi, K. S. (2004). The impact of visit frequency on the relationship between service quality and outpatient satisfaction: a South Korean study. Health services research, 39(1), 13-34.

Forster, A. J., Stiell, I., Wells, G., Lee, A. J., \& Van Walraven, C. (2003). The effect of hospital occupancy on emergency department length of stay and patient disposition. Academic Emergency Medicine, 10(2), 127-133.

Gill, J. M., Mainous III, A. G., \& Nsereko, M. (2000). The effect of continuity of care on emergency department use. Archives of family medicine, 9(4), 333.

Gilmartin, J. (2004). Day surgery: patients' perceptions of a nurse-led preadmission clinic. Journal of clinical nursing, 13(2), 243-250.

Kennelly, B., O'Callaghan, M., Coughlan, D., Cullinan, J., Doherty, E., Glynn, L., ... \& Queally, M. (2020). The COVID-19 pandemic in Ireland: An overview of the health service and economic policy response. Health Policy and Technology, 9(4), 419-429. 
Needleman, J., Buerhaus, P., Pankratz, V. S., Leibson, C. L., Stevens, S. R., \& Harris, M. (2011). Nurse staffing and inpatient hospital mortality. New England Journal of Medicine, 364(11), 1037-1045.

Robinson, A., \& Thomson, R. (2001). Variability in patient preferences for participating in medical decision making: implication for the use of decision support tools. BMJ Quality \& Safety, 10(suppl 1), i34-i38.

Saaty, T. L., \& Peniwati, K. (2013). Group decision making: drawing out and reconciling differences. RWS publications.

Sitzia, J., \& Wood, N. (1997). Patient satisfaction: a review of issues and concepts. Social science \& medicine, 45(12), 1829-1843.

Sudore, R. L., \& Fried, T. R. (2010). Redefining the "planning" in advance care planning: preparing for end-of-life decision making. Annals of internal medicine, 153(4), 256261.

Sun, N., Wei, L., Shi, S., Jiao, D., Song, R., Ma, L., ... \& Wang, H. (2020). A qualitative study on the psychological experience of caregivers of COVID-19 patients. American journal of infection control, 48(6), 592-598.

Thekkur, P., Takarinda, K. C., Timire, C., Sandy, C., Apollo, T., Kumar, A., ... \& Harries, A. D. (2021). Operational research to assess the real-time impact of COVID-19 on TB and HIV services: The experience and response from health facilities in Harare, Zimbabwe. Tropical Medicine and Infectious Disease, 6(2), 94.

Van Zyl, D. G., \& Rheeder, P. (2008). Survey on knowledge and attitudes regarding diabetic inpatient management by medical and nursing staff at Kalafong Hospital. Journal of Endocrinology, Metabolism and Diabetes in South Africa, 13(3), 90-97.

Weller, W. E., Minkovitz, C. S., \& Anderson, G. F. (2003). Utilization of medical and healthrelated services among school-age children and adolescents with special health care needs (1994 National Health Interview Survey on Disability [NHIS-D] Baseline Data). Pediatrics, 112(3), 593-603 